\section{JTI}

JOURNAL OF

TRAUMA AND INJURY

\title{
Early Predictive Values for Severe Rhabdomyolysis in Blunt Trauma
}

\author{
Jung Yun Park, M.D., Myoung Jun Kim, M.D., Jae Gil Lee, M.D., Ph.D. \\ Department of Surgery, Yonsei University College of Medicine, Seoul, Korea
}

Purpose: Rhabdomyolysis (RB) is a syndrome characterized by the decomposition of striated muscles and leakage of their contents into the bloodstream. Acute kidney injury (AKI) is the most significant and serious complication of $\mathrm{RB}$ and is a major cause of mortality in patients with RB. Severe RB (creatine kinase $[C K] \geq 5,000$ ) has been associated with AKI. However, early prediction is difficult because CK can reach peak levels 1-3 days after the trauma. Hence, the aim of our study was to identify predictors of severe RB using initial patient information and parameters.

Methods: We retrospectively analyzed 1,023 blunt trauma patients admitted to a single tertiary hospital between August 2011 and March 2018. Patients with previously diagnosed chronic kidney disease were excluded from the study. RB and severe RB were defined as a CK level $\geq 1,000 \mathrm{U} / \mathrm{L}$ and $\geq 5,000 \mathrm{U} / \mathrm{L}$, respectively. The diagnosis of AKI was based on RIFLE criteria.

Results: The overall incidence of RB and severe RB was 31.3\% $(\mathrm{n}=320)$ and $6.2 \%(\mathrm{n}=63)$, respectively. On multivariable analysis, male sex (odds ratio [OR] 3.78, 95\% confidence interval [CI] 1.43 to 10.00 ), initial base excess (OR $0.85,95 \%$ CI 0.80 to 0.90 ), initial CK (OR 2.07, 95\% CI 1.67 to 2.57), and extremity abbreviated injury scale score (OR 1.78, $95 \%$ CI 1.39 to 2.29) were found to predict severe RB. The results of receiver operating characteristic analysis showed that the best cutoff value for the initial serum CK level predictive of severe RB was 1,494 U/L.

Conclusions: Male patients with severe extremity injuries, low base excess, and initial CK level $>1,500 \mathrm{U} / \mathrm{L}$ should receive vigorous fluid resuscitation.

Keywords: Rhabdomyolysis; Creatine kinase; Acute kidney injury

\section{INTRODUCTION}

Rhabdomyolysis $(\mathrm{RB})$ is a syndrome characterized by the decomposition of striated muscles and leakage of their contents into the bloodstream, including electrolytes, myoglobin, and other sarcoplasmic proteins (e.g., creatine kinase [CK], lactate dehydrogenase, alanine aminotransferase, and aspartate aminotransferase) [1]. The (http://creativecommons.org/licenses/by-nc/4.0/) which permits unrestricted noncommercial use, distribution, and reproduction in any medium, provided the original work is properly cited. 
presentation of $\mathrm{RB}$ ranges from asymptomatic elevation of serum CK to life-threatening conditions such as acute kidney injury (AKI), cardiac arrhythmias, compartment syndrome, hypovolemic shock, and disseminated intravascular coagulation $[1,2]$.

AKI is the most significant and serious complication of RB and major cause of mortality among RB patients [1]. Myoglobin plays a major role in the pathogenesis of RB-induced AKI. However, myoglobin is not an appropriate diagnostic marker. Because of its short half-life (2-3 hours), myoglobin is rapidly metabolized and excreted [3]. The most sensitive biomarker of muscle injury is the serum CK level. The diagnosis of RB is generally made when CK levels are five times the upper normal limit $(>1,000 \mathrm{U} / \mathrm{L})$ in the presence of skeletal muscle injury $[4,5]$. CK is not directly involved in the pathogenesis of myoglobinuric AKI, but CK levels $>5.000 \mathrm{U} / \mathrm{L}$ indicate serious muscle injury and are associated with the onset of AKI and mortality $[3,6]$.

The most common cause of RB is trauma accompanied by muscle damage $[3,4,7]$. Myoglobinuric AKI can be prevented with timely and vigorous fluid resuscitation. However, recent studies have shown that large volumes of fluid could worsen the outcome in view of coagulopathy and hypothermia [8]. Therefore, it is important to identify the group of trauma patients at high risk for AKI. Numerous studies have evaluated the risk factors for AKI in RB patients. Most have shown that the peak CK level is associated with AKI $[2,6,9]$. However, CK can reach peak levels 1-3 days after trauma [3]. Hence, the aim of our study was to identify predictors of severe RB on the basis of initial patient information and parameters.

\section{METHODS}

\section{Patient enrollment and data collection}

We conducted a retrospective observational study of blunt trauma patients admitted to a single tertiary hospital in Seoul, Korea between August 2011 and March 2018. We selected all subjects who had visited ER after trauma during our study period, and excluded them using following criteria: 1) patients who did not undergo blood CK level test, 2) no blunt trauma, 3) patients who discharged or transferred out at ER, 4) patients with known chronic kidney disease (CKD), 5) patients who transferred from another hospital in chronic phase, and 6) patients who died within first 48 hours after arriving to the ER. Data on patient ages, sex, injury severity score (ISS), and abbreviated injury scale (AIS) score were extracted from electronic files. Initial blood pressure, heart rate, $\mathrm{pH}$, base excess $(\mathrm{BE})$, lactate, and hemoglobin $(\mathrm{Hb})$ values were analyzed at the time of presentation to the hospital. Peak CK levels were determined within 72 hours of trauma. Given that the diagnosis of $\mathrm{RB}$ is made when the $\mathrm{CK}$ levels are five times the upper normal limit $(1,000 \mathrm{U} / \mathrm{L})$, we considered $5,000 \mathrm{U} / \mathrm{L}$ as the cut-off in the diagnosis of severe RB $[3,6]$. Patients previously diagnosed with chronic kidney disease and those who died during the first 48 hours after presentation were excluded (Fig. 1).

\section{Clinical variables}

The acute dialysis quality initiative criteria (risk, injury, and failure, and loss, and end-stage kidney disease; RIFLE classification) were used to classify AKI. The RIFLE classification defines three grades of increasing severity of AKI, that is, risk (R), injury (I), and failure (F), as well as two outcome variables, that is, loss $(\mathrm{L})$ and end-stage kidney disease (E) (Fig. 2) [10]. We calculated the baseline creatinine level of patients without CKD from the Modification of Diet in Renal Disease equation using an estimated glomerular filtration rate of $75 \mathrm{~mL} / \mathrm{min} / 1.73 \mathrm{~m}^{2}$, similar to previous studies [11]. For analysis, patients were assigned to their worst RIFLE category according to serum creatinine criteria during the first 7 days after admission.

\section{Statistical analysis}

Categorical variables were analyzed using the $\chi^{2}$ test or Fisher's exact test, as appropriate. Continuous variables were analyzed using Student's $t$-test. A $p$-value less than 0.05 was considered statistically significant. A multiple logistic regression model was then developed using the variables that were determined to be significantly associated with severe RB. Distributions of variables were reported as mean \pm standard deviation (SD) (percentages) and median (interquartile range). Receiver operating characteristic (ROC) analysis was used to evaluate the appropriate cutoff points of the biomarkers and to assess 


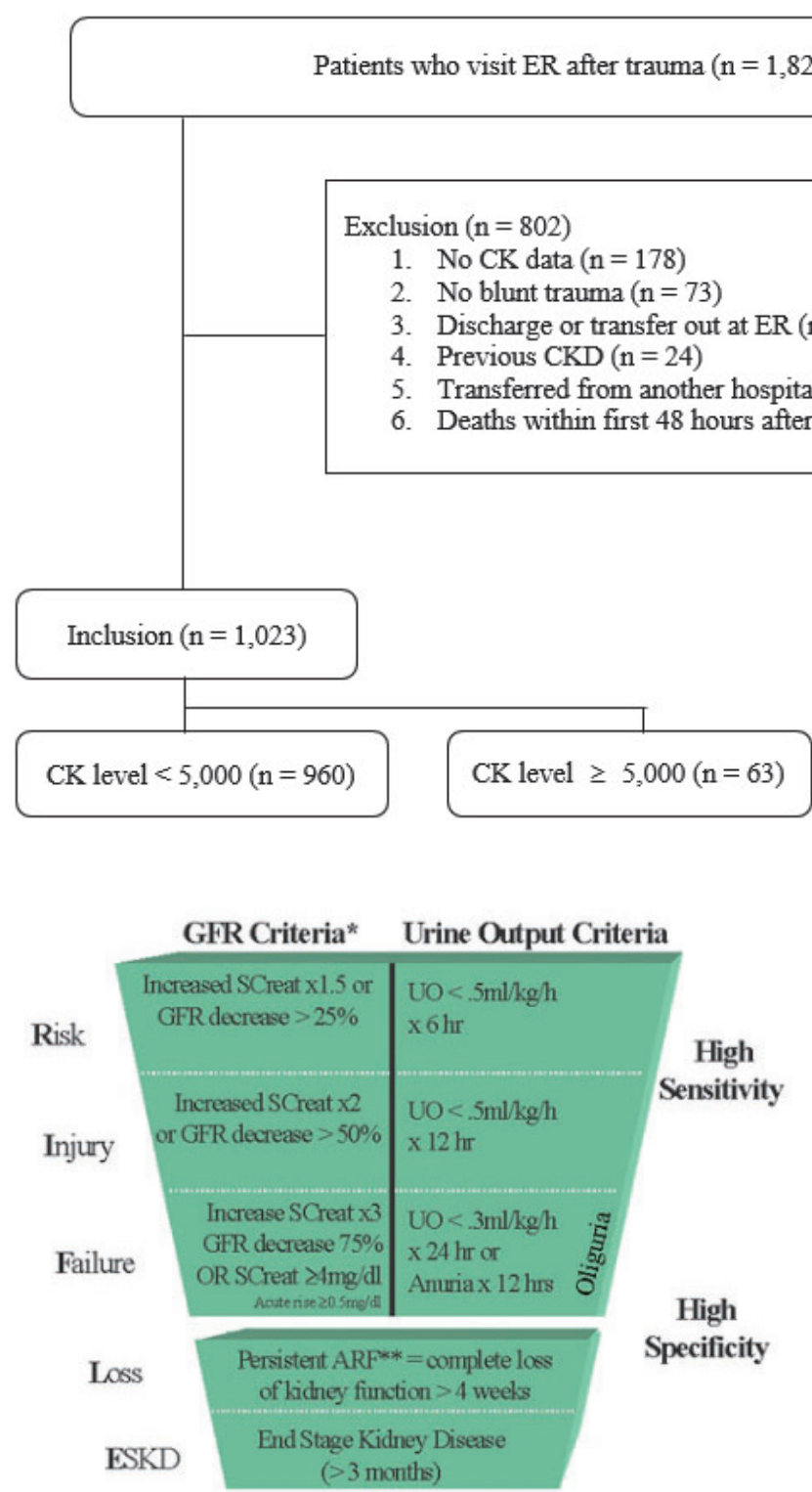

Fig. 2. Classification scheme of RIFLE criteria adapted from reference [10]. GFR: glomerular filtration rate, UO: urine output, ARF: acute renal failure, ESKD: end stage kidney disease, RIFLE: risk, injury, and failure, loss, and end-stage kidney disease.

the sensitivity and specificity of the predictors for severe RB. The test characteristics of these different cutoff values, including sensitivity, specificity, and area under the ROC curve (AUC), were determined. Statistical analyses were performed with IBM SPSS software (version 23.0; IBM, Armonk, NY, USA).
Fig. 1. Flow diagram of article selection and the inclusion/exclusion process. ER: emergency room, CK: creatine kinase, CKD: chronic kidney disease.

\section{RESULT}

\section{Initial clinical and laboratory variables}

During the study period (August 2011 to March 2018), we analyzed 1,023 patients (729 males and 294 females; age, $47.7 \pm 19.6$, mean \pm SD) admitted with blunt trauma, who met the criteria. Of 63 patients with severe RB, 30 (47.6\%) developed AKI, 16 (25.4\%) needed renal replacement therapy, and nine (14.3\%) died within 30 hospital days (Table 1). Patients with severe RB were more likely to be male and to have a significantly lower systolic blood pressure. They were also noted to have higher ISS, especially extremity AIS scores. There was no difference in the head \& neck AIS scores (2 [0-3] vs. $2[0-3], p=0.822)$ (Table 2).

When comparing initial laboratory values between the groups (with or without severe RB), indicators of metabolic acidosis, including $\mathrm{pH}$, lactate, $\mathrm{BE}$, and bicarbonate showed significant differences. CK, creatinine, and most ions, including $\mathrm{Mg}^{2+}, \mathrm{K}^{+}$, and $\mathrm{P}^{-}$were higher in the severe $\mathrm{RB}$ group, whereas $\mathrm{Ca}^{2+}$ and $\mathrm{Hb}$ were lower (Table 3 ).

\section{Multivariable logistic regression and ROC analysis of risk factors for severe RB}

Using the variables that showed statistically significant results in Tables 2 and 3, multivariable logistic regression 
Table 1. Outcomes according to RB and sRB

\begin{tabular}{|c|c|c|c|c|c|}
\hline & Total $(n=1,023)$ & $R B(n=320)$ & $p$-value & sRB $(n=63)$ & $p$-value \\
\hline Emergency Op & $239(23.4)$ & 97 (30.3) & $\leq 0.001$ & 25 (39.7) & 0.002 \\
\hline AKI & $103(10.1)$ & $69(21.6)$ & $\leq 0.001$ & $30(47.6)$ & $\leq 0.001$ \\
\hline RRT & $30(2.9)$ & $27(8.4)$ & $\leq 0.001$ & $16(25.4)$ & $\leq 0.001$ \\
\hline 30-day mortality & $39(3.8)$ & $27(8.4)$ & $\leq 0.001$ & $9(14.3)$ & $\leq 0.001$ \\
\hline H-LOS & $15(8-29)$ & $20(12-39)$ & 0.016 & $26(14-47)$ & 0.049 \\
\hline ICU-LOS & $1(0-6)$ & $3(1-11)$ & 0.087 & $5(2-19)$ & 0.234 \\
\hline
\end{tabular}

Values are presented as median (interquartile range) or numer (\%).

RB: rhabdomyolysis, sRB: severe rhabdomyolysis, Op: operation, AKl: acute kidney injury, RRT: renal replacement therapy, H-LOS: hospital length of stay,

ICU-LOS: intensive care unit length of stay.

Table 2. Characteristics according to sRB

\begin{tabular}{|c|c|c|c|c|}
\hline & Total $(n=1,023)$ & Non sRB $(n=960)$ & $\operatorname{sRB}(n=63)$ & $p$-value \\
\hline Age & $47.7 \pm 19.6$ & $47.4 \pm 19.8$ & $43.2 \pm 18.0$ & 0.101 \\
\hline Male & 729 (71.3) & $673(70.1)$ & $56(88.9)$ & 0.001 \\
\hline Initial GCS & $13.3 \pm 3.5$ & $13.4 \pm 3.5$ & $12.5 \pm 4.2$ & 0.103 \\
\hline Initial SBP & $122.8 \pm 32.7$ & $124.3 \pm 32.0$ & $102.3 \pm 36.2$ & $\leq 0.001$ \\
\hline Initial HR & $90.2 \pm 22.2$ & $88.9 \pm 21.8$ & $98.5 \pm 28.3$ & 0.010 \\
\hline Initial BT & $36.7 \pm 4.8$ & $36.7 \pm 4.6$ & $36.1 \pm 1.4$ & 0.315 \\
\hline ISS & $18 \pm 11$ & $18 \pm 11$ & $25 \pm 12$ & $\leq 0.001$ \\
\hline Head \& neck AIS & $2(0-3)$ & $2(0-3)$ & $2(0-3)$ & 0.822 \\
\hline Face AlS & $0(0-1)$ & $0(0-1)$ & $0(0-2)$ & 0.186 \\
\hline Chest AIS & $1(0-3)$ & $1(0-3)$ & $2(0-3)$ & 0.024 \\
\hline Abdomen AIS & $0(0-2)$ & $0(0-2)$ & $2(0-3)$ & 0.020 \\
\hline Extremity AIS & $2(0-2)$ & $2(0-2)$ & $3(2-3)$ & $\leq 0.001$ \\
\hline External AIS & $1(0-1)$ & $1(0-1)$ & $1(0-1)$ & 0.102 \\
\hline
\end{tabular}

Values are presented as mean \pm standard deviation, median (interquartile range), or number (\%).

RB: rhabdomyolysis, sRB: severe rhabdomyolysis, GCS: Glasgow coma scale, SBP: systolic blood pressure, HR: heart rate, BT: body temperature, ISS: injury severity score, AlS: abbreviated injury scale.

analysis was performed (Table 4). Male sex (odds ratio [OR] 3.78, 95\% confidence interval [CI] 1.43 to 10.00$)$, BE (OR 0.85, 95\% CI 0.80 to 0.90 ), CK (OR 2.07, 95\% CI 1.67 to 2.57 ), and extremity AIS scores (OR 1.78, 95\% CI 1.39 to 2.29) were predictive of severe $\mathrm{RB}$. As the $\mathrm{CK}$ range was very wide, 1/1,000 values were used for analysis. ROC analysis showed that the AUC for the initial serum CK level predictive of severe RB was 0.80 (Fig. 3) and that the best cutoff value for the initial serum $\mathrm{CK}$ level predictive of severe RB was 1,494 U/L (sensitivity, 0.46; specificity, $0.90)$.

\section{DISCUSSION}

The mortality rate of $\mathrm{RB}$ varies widely according to the study population, cause of RB, and diagnostic cutoff point for RB [1,3]. Veenstra et al. [12], in a study of 93 patients with severe $\mathrm{RB}$, reported mortality of $32 \%$ and an $\mathrm{AKI}$ incidence of $51 \%$. In this study, the 30 -day mortality rate was significantly higher in the severe RB group, and the incidence of AKI was similar to that in the previous study. Safari et al. [6], in a recent meta-analysis that evaluated the relationship between serum CK and the development 
Table 3. Univariable analysis of initial laboratory values according to sRB

\begin{tabular}{|c|c|c|c|c|}
\hline & Total $(n=1,023)$ & Non-sRB $(n=960)$ & $s R B(n=63)$ & $p$-value \\
\hline $\mathrm{pH}$ & $7.35 \pm 0.11$ & $7.36 \pm 0.10$ & $7.23 \pm 0.17$ & $\leq 0.001$ \\
\hline Lactate $(\mathrm{mmol} / \mathrm{L})$ & $3.3 \pm 2.8$ & $3.1 \pm 2.6$ & $6.5 \pm 4.4$ & $\leq 0.001$ \\
\hline $\mathrm{BE}(\mathrm{mmol} / \mathrm{L})$ & $-6.2 \pm 4.5$ & $-5.8 \pm 4.2$ & $-11.1 \pm 5.6$ & $\leq 0.001$ \\
\hline Bicarbonate (mmol/L) & $19.3 \pm 3.4$ & $19.6 \pm 3.2$ & $16.0 \pm 3.9$ & $\leq 0.001$ \\
\hline PCO2 $(\mathrm{mmHg})$ & $37.6 \pm 11.5$ & $36.9 \pm 10.3$ & $47.8 \pm 20.9$ & $\leq 0.001$ \\
\hline $\mathrm{PO} 2(\mathrm{mmHg})$ & $96.8 \pm 41.5$ & $97.4 \pm 41.4$ & $87.2 \pm 41.7$ & 0.059 \\
\hline Calcium (mg/dL) & $4.50 \pm 0.33$ & $4.51 \pm 0.32$ & $4.20 \pm 0.42$ & $\leq 0.001$ \\
\hline Magnesium (mg/dL) & $1.46 \pm 0.25$ & $1.46 \pm 0.25$ & $1.55 \pm 0.25$ & 0.004 \\
\hline Potassium (mmol/L) & $4.2 \pm 0.6$ & $4.1 \pm 0.5$ & $4.6 \pm 0.7$ & $\leq 0.001$ \\
\hline Phosphorus (mg/dL) & $3.7 \pm 1.3$ & $3.6 \pm 1.2$ & $4.8 \pm 2.2$ & $\leq 0.001$ \\
\hline $\mathrm{Hb}(\mathrm{g} / \mathrm{dL})$ & $12.2 \pm 2.6$ & $12.4 \pm 2.6$ & $10.9 \pm 2.8$ & $\leq 0.001$ \\
\hline $\mathrm{Cr}(\mathrm{mg} / \mathrm{dL})$ & $0.9 \pm 0.3$ & $0.9 \pm 0.3$ & $1.2 \pm 0.5$ & $\leq 0.001$ \\
\hline $\mathrm{BUN}(\mathrm{mg} / \mathrm{dL})$ & $16.6 \pm 6.9$ & $16.4 \pm 6.8$ & $19.0 \pm 6.3$ & 0.004 \\
\hline CK (U/L) & $300(171-779)$ & $284(165-668)$ & $1,101(485-5,750)$ & $\leq 0.001$ \\
\hline
\end{tabular}

Values are presented as mean \pm standard deviation or median (interquartile range).

sRB: severe rhabdomyolysis, RB: rhabdomyolysis, BE: base excess, Hb: hemoglobin, Cr: creatinine, BUN: blood urea nitrogen, CK: creatine kinase.

Table 4. Multivariable logistic regression analysis of risk factors for SRB

\begin{tabular}{|lccc|}
\hline Variable & OR & 95\% Cl & p-value \\
\hline Male & 3.777 & $1.426-10.000$ & 0.007 \\
BE & 0.846 & $0.796-0.899$ & $\leq 0.001$ \\
CK & 2.074 & $1.674-2.571$ & $\leq 0.001$ \\
Extremity AIS & 1.782 & $1.388-2.289$ & $\leq 0.001$ \\
\hline
\end{tabular}

sRB: severe rhabdomyolysis, OR: odds ratio, Cl: confidence interval, BE: base excess, CK: creatine kinase, AlS: abbreviated injury scale.

${ }^{a}$ As the CK range was very wide, 1/1,000 values were used for analysis.

of RB-induced AKI, showed a significant association between the mean serum CK level and risk for AKI [6]. However, as cutoff points ranged from 1,000 to 15,000 U/L in prior studies, the hazardous cutoff point for $\mathrm{CK}$ in the development of AKI remains unclear. Furthermore, because the peak value develops during the hospital stay, the CK level presented in previous studies cannot be used to predict the risk of AKI in the early stage.

In this study, male sex, BE, CK level, and extremity AIS score were found to be early predictors of severe RB. In particular, the AUC for initial CK was considered to be

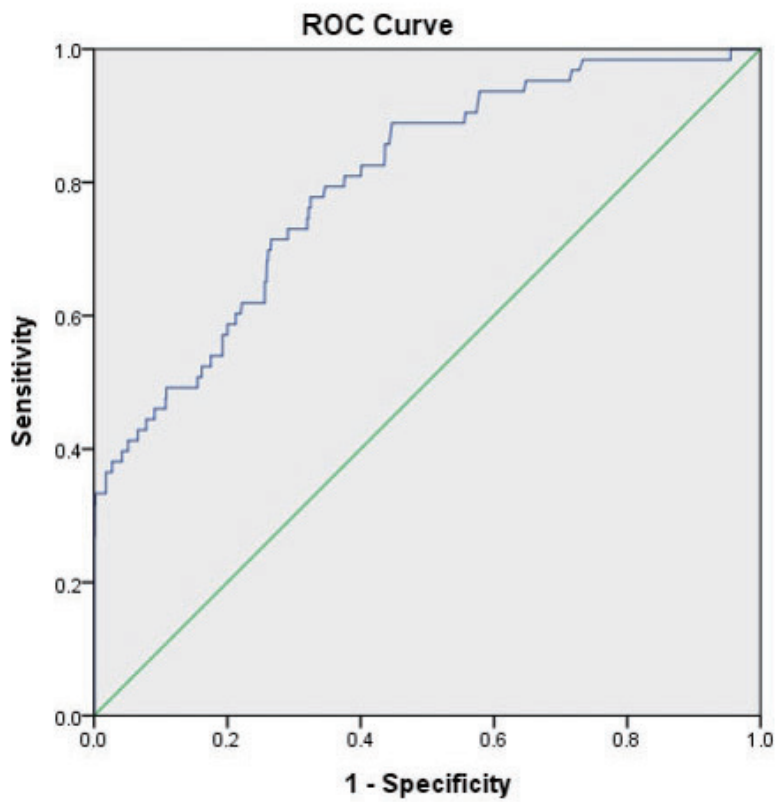

Fig. 3. Receiver operating characteristic curve for initial serum creatine kinase in predicting severe rhabdomyolysis caused by blunt trauma. ROC: receiver operating characteristic.

"good" at predicting severe RB. The specificity is more important in setting the cutoff point for the CK level, as 
previous studies have suggested higher prophylactic volume infusions of up to 4-6 L per day. Therefore, we suggest $1,500 \mathrm{U} / \mathrm{L}$ (specificity $>0.90$ ) as the initial CK cutoff value for severe RB.

Several limitations of the present study should be considered. The results were based on a retrospective, single-center review of medical records. Of the 1,023 patients, only 63 patients met criteria for severe $\mathrm{RB}$, and it is relatively small sample size. Details of the trauma mechanism may not have been rigorously documented, and not all serologic tests were performed in all patients. In addition, the basal creatinine level was calculated as a glomerular filtration rate of 0.75 for all patients without CKD because the basal creatinine level was unknown in the majority of patients. Consequently, the actual incidence of AKI might be different. These limitations may lead to some bias in analyzing the factors associated with severe RB and AKI.

\section{CONCLUSION}

AKI is the most significant and serious complication of $\mathrm{RB}$ and major cause of mortality among RB patients. Severe RB has been strongly associated with the onset of AKI and mortality. Male sex, initial BE, initial serum levels of CK, and extremity AIS score can be key predictors of severe RB that can lead to AKI. We suggest that male patients with severe extremity injury, low $\mathrm{BE}$, and initial CK level $>1,500 \mathrm{U} / \mathrm{L}$ should receive vigorous fluid resuscitation.

\section{REFERENCES}

1. Bosch X, Poch E, Grau JM. Rhabdomyolysis and acute kidney injury. N Engl J Med 2009;361:62-72.

2. Rodríguez E, Soler MJ, Rap O, Barrios C, Orfila MA, Pascual J. Risk factors for acute kidney injury in severe rhabdomyolysis.
PLoS One 2013;8:e82992.

3. Huerta-Alardín AL, Varon J, Marik PE. Bench-to-bedside review: Rhabdomyolysis -- an overview for clinicians. Crit Care 2005;9:158-69.

4. Baeza-Trinidad R, Brea-Hernando A, Morera-Rodriguez S, Brito-Diaz Y, Sanchez-Hernandez S, El Bikri L, et al. Creatinine as predictor value of mortality and acute kidney injury in rhabdomyolysis. Intern Med J 2015;45:1173-8.

5. Zimmerman JL, Shen MC. Rhabdomyolysis. Chest 2013;144: 1058-65.

6. Safari S, Yousefifard M, Hashemi B, Baratloo A, Forouzanfar MM, Rahmati F, et al. The value of serum creatine kinase in predicting the risk of rhabdomyolysis-induced acute kidney injury: a systematic review and meta-analysis. Clin Exp Nephrol 2016;20:153-61.

7. Chen CY, Lin YR, Zhao LL, Yang WC, Chang YJ, Wu HP. Clinical factors in predicting acute renal failure caused by rhabdomyolysis in the ED. Am J Emerg Med 2013;31:1062-6.

8. Jacob M, Kumar P. The challenge in management of hemorrhagic shock in trauma. Med J Armed Forces India 2014;70:163-9.

9. Simpson JP, Taylor A, Sudhan N, Menon DK, Lavinio A. Rhabdomyolysis and acute kidney injury: creatine kinase as a prognostic marker and validation of the McMahon Score in a 10-year cohort: a retrospective observational evaluation. Eur J Anaesthesiol 2016;33:906-12.

10. Bellomo R, Ronco C, Kellum JA, Mehta RL, Palevsky P; Acute Dialysis Quality Initiative workgroup. Acute renal failure - definition, outcome measures, animal models, fluid therapy and information technology needs: the second international consensus conference of the Acute Dialysis Quality Initiative (ADQI) group. Crit Care 2004;8:R204-12.

11. Delaney KA, Givens ML, Vohra RB. Use of RIFLE criteria to predict the severity and prognosis of acute kidney injury in emergency department patients with rhabdomyolysis. J Emerg Med 2012;42:521-8.

12. Veenstra J, Smit WM, Krediet RT, Arisz L. Relationship between elevated creatine phosphokinase and the clinical spectrum of rhabdomyolysis. Nephrol Dial Transplant 1994;9:637-41. 